\title{
Common and specific downstream signaling targets controlled by TIr2 and Tlr5 innate immune signaling in zebrafish
}

Shuxin Yang ${ }^{1}$, Rubén Marín-Juez ${ }^{2,3}$, Annemarie H. Meijer ${ }^{1}$ and Herman P. Spaink ${ }^{1 *}$ (D)

\begin{abstract}
Background: Although the responses to many pathogen associated molecular patterns (PAMPs) in cell cultures and extracted organs are well characterized, there is little known of transcriptome responses to PAMPs in whole organisms. To characterize this in detail, we have performed RNAseq analysis of responses of zebrafish embryos to injection of PAMPs in the caudal vein at one hour after exposure. We have compared two ligands that in mammals have been shown to specifically activate the TLR2 and TLR5 receptors: Pam3CSK4 and flagellin, respectively.

Results: We identified a group of 80 common genes that respond with high stringency selection to stimulations with both PAMPs, which included several well-known immune marker genes such as illb and tnfa. Surprisingly, we also identified sets of 48 and 42 genes that specifically respond to either Pam3CSK4 or flagellin, respectively, after a comparative filtering approach. Remarkably, in the Pam3CSK4 specific set, there was a set of transcription factors with more than 2 fold-change, as confirmed by qPCR analyses, including cebpb, fosb, nr4a 1 and egr3. We also showed that the regulation of the Pam3CSK4 and flagellin specifically responding sets is inhibited by knockdown of t/r2 or t/r5, respectively.
\end{abstract}

Conclusions: Our studies show that Pam3CSK4 and flagellin can stimulate the TIr2 and TIr5 signaling pathways leading to common and specific responses in the zebrafish embryo system.

Keywords: TLR signaling, Zebrafish, TLR2, TLR5, Flagellin, Pam3CSK4, Innate immunity

\section{Background}

The innate immune system is referred to as the first line in host defense against invading pathogens $[1,2]$. Its highly developed ability to recognize microbial patterns and host-derived danger signals relies on so-called pattern recognition receptors (PRRs), especially on the Tolllike receptors (TLRs) [3-5]. In humans, the TLR family is composed of 10 members, which are located at the cell surface with the exception of TLR3, 7, 8 and 9, which are localized on intracellular endosomal membranes [6-8]. The TLRs are involved in the recognition of a wide variety of ligands, including pathogen associated molecular patterns (PAMPs), such as bacterial cell wall components and viral RNA, as well as damage associated molecular patterns (DAMPs) $[9,10]$. This leads to

\footnotetext{
* Correspondence: h.p.spaink@biology.leidenuniv.nl

${ }^{1}$ Institute of Biology, Leiden University, PO Box 95022300 RA Leiden, The Netherlands

Full list of author information is available at the end of the article
}

subsequent intracellular signal transduction, triggering the production of inflammatory cytokines and chemokines, but it can also lead to anti-inflammatory responses as has been recently shown for TLR10 acting as a heterodimer with TLR2 [11, 12].

The recognition of PAMPs and DAMPs by different TLRs is directed by structurally conserved leucine-rich repeats (LRR) motifs of the TLRs ectodomains (ECDs) [13, 14]. Cell-surface TLRs can mediate binding to PAMPs by homodimerizing, like TLR4 and TLR5 that recognize lipopolysaccharide (LPS) [15] and flagellin [16], respectively. In contrast, other TLRs form heterodimers like TLR2 that associates with TLR1, TLR6 or TLR10, in conjunction recognizing lipopeptides and lipoproteins $[12,17,18]$. The diversity of TLR2 dimer combinations is thought to be responsible for its extensive recognition ability, ranging from the diverse components of various pathogens to the host heat-shock protein 70 [10]. TLR2 plays an important role in resistance to the infection induced by 
Mycobacterium tuberculosis $(M t b)$ [19, 20]. For example, McBride et al. demonstrated that TLR2 knockout mice show enhanced cell infiltration and inflammation in lungs upon low dose chronic infection with $M t b$, and fail to stably control the bacterial burden [21]. A series of components of $M t b$ can trigger the TLR2 signaling pathway upon infection, such as triacylated lipoprotein LprG, LpqH and PhoS1 [22, 23], and glycolipid lipoarabinomannan [24]. Pam3CysSerLys4 (Pam3CSK4) is a synthetic triacylated lipopeptide that mimics the triacylated lipoprotein of mycobacteria and classical gram-positive bacteria, which can be recognized by TLR2/TLR1 heterodimers and induce the production and release of pro- and antiinflammatory cytokines (IL-6, IL-12, TNF- $\alpha$ and IL-10), chemokines (IL-8) and interferon (IFN- $\gamma$ ) [25-28]. Most of these studies on the responses of the TLR2 signaling pathway have been performed in cell culture systems. A notable exception is the reported transcriptome response of mouse mononuclear phagocytes recruited to lungs challenged with Pam3CSK4 as measured by micro-arrays [29]. As another example 8 day old mice were treated with Pam3CSK4 and analyzed for the expression of several inflammatory genes using qPCR [30].

The zebrafish embryonic model has much potential to study the ligand specificity of TLRs at the organism level [29]. Importantly, zebrafish offers the possibility to study the innate immune system separated from the adaptive immune system in their embryonic and larval stages (up to 3-4 weeks post fertilization) [31-33]. To date, a number of TLR signaling pathway mediators have been identified and studied in zebrafish such as the adaptor proteins Myd88, Tirap (Mal), Trif and Sarm1 [34-38], and the downstream signaling intermediates Irak and Traf6 [39, 40].

In work previously published by our group, we demonstrated that the function and regulation of the zebrafish homologs of human TLR5, $t \operatorname{lr} 5 a$ and $t l r 5 b$, are conserved with their mammalian counterparts. Both $t \operatorname{lr} 5 a$ and $t \operatorname{lr} 5 b$ are strongly up-regulated in response to Salmonella typhimurium infection [41]. Furthermore, in the same study it was shown that knockdown of these two genes prevented or weakened the activation of genes for several inflammatory mediators like $m m p 9, \mathrm{cxcl}-\mathrm{Clc}$, $i l 1 b$ and il8 upon flagellin stimulation [41].

In this study, we aimed to study TLR2 function, in comparison with TLR5, in zebrafish using transcriptome analysis. Injection into the blood stream of the $t \operatorname{lr} 2 / \operatorname{tr} 1$ ligand Pam3CSK4, was followed by transcriptome profiling to characterize key genes involved in the early response to this PAMPs. In addition, by comparing the transcriptome response towards treatment with flagellin, we were able to discriminate non-specific immune responsive genes from a set of genes which are regulated by $t$ lr 2 but not by $t \operatorname{lr} 5$.

\section{Methods}

\section{Zebrafish husbandry}

Wild-type zebrafish of the AB/TL strain were handled in compliance with the local animal welfare regulations and maintained according to standard protocols (zfin. org). Embryos were raised in egg water $(60 \mathrm{~g} / \mathrm{ml}$ Instant Ocean sea salts) at $28.5{ }^{\circ} \mathrm{C}$. For the duration of bacterial injections, embryos were kept under anesthesia in egg water containing $0.02 \%$ buffered 3-aminobenzoic acid ethyl ester (Tricaine). The breeding of adult fish was approved by the local animal welfare committee (DEC) of the University of Leiden. All protocols adhered to the international guidelines specified by the EU Animal Protection Directive 2010/63/EU.

\section{Morpholino injections}

Morpholino oligonucleotides (Gene Tools) were diluted to desired concentrations in $1 \times$ Danieu's buffer $(58 \mathrm{mM}$ $\mathrm{NaCl}, 0.7 \mathrm{mM} \mathrm{KCl}, 0.4 \mathrm{mM} \mathrm{MgSO}, 0.6 \mathrm{mM} \mathrm{Ca}\left(\mathrm{NO}_{3}\right)_{2}$, $5.0 \mathrm{mM}$ HEPES $(\mathrm{pH}$ 7.6)) containing $1 \times$ phenol red (Sigma-Aldrich). For knockdown experiments, tlr2 ATG-morpholino (tlr2 mo, Additional file 1: Table S1) was injected with the optimal concentration at $0.5 \mathrm{mM}$ and $1 \mathrm{nl}$ volume per embryo at $0 \sim 2$ cell stage. $\operatorname{tlr} 5$ translation was blocked via injecting $1 \mathrm{nl}$ of the $t \operatorname{lr} 5 a$ and $t \operatorname{lr} 5 b$ ATG-morpholinos at a dose of $0.1 \mathrm{mM}$ and $0.5 \mathrm{mM}$ at $0 \sim 2$ cell stage, as previously published by Stockhammer and coworkers [41]. Control embryos were injected with the standard control morpholino (Sc mo, Additional file 1: Table S1).

\section{Ligands injection}

For the ligands injection assay, purified Pam3CSK4 (InvivoGen) and flagellin from S. typhimurium (Flagellin FliC VacciGrade $^{\mathrm{Tx}}$, Invitrogen) were respectively diluted to $1 \mathrm{mg} / \mathrm{ml}$ and $100 \mu \mathrm{g} / \mathrm{ml}$ in sterile water. For their administration, $1 \mathrm{nl}$ of the ligands was injected into the blood stream at $27 \mathrm{hpf}$, and sterile water was used as control. Injections were perormed using a FemtoJet microinjector (Eppendorf) and a micromanipulator with pulled microcapillary pipettes.

\section{RNA isolation, cDNA synthesis and qPCR}

RNA was extracted using TRIzol Reagent (Life Technologies) and purified by column according to the manufacturer's instructions of RNeasy MinElute Cleanup Kit (Qiagen). The concentration and quality of RNA were detected by NANODROP 2000/2000c (Thermo Scientific). $1 \mu \mathrm{g}$ cDNA synthesis reactions and qPCR were performed as described in the manufacturer's instructions (iScript ${ }^{\mathrm{TM}}$ cDNA Synthesis Kit and $\mathrm{iQ}^{\mathrm{TM}} \mathrm{SYBR}^{\odot}$ Green Supermix, BioRad) and normalized against the expression of ppial as a housekeeping gene [42]. PCR analysis was performed using the following protocol: $95{ }^{\circ} \mathrm{C} 3 \mathrm{~min}, 40$ cycles real 
time of $95^{\circ} \mathrm{C} 15 \mathrm{~s}$ and $60{ }^{\circ} \mathrm{C} 45 \mathrm{~s}$, and final melting curve of 81 cycles from $95{ }^{\circ} \mathrm{C} 1 \mathrm{~min}$ to $55^{\circ} \mathrm{C} 10 \mathrm{~s}$. Results were analyzed using the $\Delta \Delta \mathrm{Ct}$ method. Primer sequences used can be found in Additional file 1: Table S1.

\section{Deep sequencing and data analyzing}

Triplicates of $10 \sim 20$ embryos of $\mathrm{AB} / \mathrm{TL}$ or tlr morphants from three injection conditions, Pam3CSK4, flagellin or water injection, were homogenized in $500 \mu \mathrm{l}$ of Trizol reagent (Qiagen). Total RNA was extracted and column-purified according to the manufacturer's instructions of the RNeasy MinElute Cleanup Kit (Qiagen). The subsequent sample preparation and Illumina RNA sequencing were as previously described [43]. RNA samples were treated with DNaseI (Life Technologies) to remove residual genomic DNA. RNA integrity was analyzed by Lab-on-a-chip analysis (Agilent, Amstelveen, The Netherlands). A total of $2 \mu \mathrm{g}$ of RNA was used to make RNAseq libraries using the Illumina TruSeq RNA Sample Preparation Kit v2 (Illumina, Inc., San Diego, CA, USA). The manufacturer's instructions were followed with the exception of two modifications. In the adapter ligation step, $1 \mu \mathrm{l}$, instead of $2.5 \mu \mathrm{l}$, adaptor was used. In the library size-selection step, the library fragments were isolated with a double Ampure XP purification with a $0.7 \times$ beads to library ratio (Beckman Coulter, Woerden, The Netherlands). The resulting mRNAseq library was sequenced using an Illumina HiSeq2500 Instrument (Illumina, Inc.) according to the manufacturer's instructions with a read length of $2 \times 50$ nucleotides. Image analysis and base-calling were done using the Illumina HCS version 2.0.12. The raw data has been submitted to the GEO database (accession number GSE64570). The total number of reads for each sample is summarized in Additional file 2: Table S4 and quality control was according to the sequencing company guidelines (ZFsceens.com). The data was analyzed using the GeneTiles software (http://www.genetiles.com) [44] using a cut-off setting of 2 fold-change and a $p$-value $<0.01$. In brief, Genetiles used fastq files as input for the program Bowtie2 [44] to align the reads to the zebrafish genome (obtained from Ensembl version Zv9). Subsequently, the programs SAMtools [45], DESeq and DEXSeq [46, 47] are used for data processing. The complete data processing pipeline for Genetiles, including the used parameters, is available for download at www.genetiles.com and can also be found in Veneman et al [44]. Using these settings we have mapped the numbers of reads as shown in Additional file 2: Table S4. The triplicate data sets of Pam3CSK4, flagellin and control treatments were mapped to 27104, 26583, and 26409 ENSEMBL genes, respectively. The difference between the mapped reads of the individual samples compared with the mapped reads of triplicate samples was always lower than $12 \%$ (Additional file 2:
Table S4). GO analysis was performed using the software package DAVID available at http://david.abcc.ncifcrf.gov/ home.jsp [48].

\section{Results}

The immune response of zebrafish embryos to injection of PAMPs in the caudal vein

In a recent study by Stockhammer et al, it was shown that flagellin injected into the caudal vein at $27 \mathrm{~h}$ post fertilization (hpf) induced several immune response marker genes as measured by qPCR [41]. To further characterize the response to another well characterized PAMP, we injected Pam3CSK4 using the same method. The expression levels of cytokine genes $i l 1 b$, tnfa and $i l 6$, the chemokine gene $i l 8$, and anti-inflammatory gene $i l 10$, were measured by qPCR at 1,3 and 6 h post injection (hpi) respectively (Fig. 1). The results show that there was a significant up-regulation of these genes upon Pam3CSK4 stimulation. For all these marker genes the induction was transient and followed by a gradual decrease over time. The il1b gene was the only marker of which up-regulation was observed at 1 and $3 \mathrm{hpi}$, with a significantly higher expression than the control group (Fig. 1a). For the tnfa, il6, il8 and il10 genes there was a more obvious decrease of induction over time (Fig. 1b-e). These results show that Pam3CSK4 induces similar responses in zebrafish as in mammalian cells [25-28] suggesting that this response is also mediated via the $t l r 2$ signaling pathway.

\section{The function of Tlr5 and Tlr2 in the immune response towards flagellin and Pam3CSK4}

In order to study the function of $t \operatorname{tr} 2$ and $t \operatorname{tr} 5$ in the above described responses to Pam3CSK4 and flagellin we used morpholinos to knockdown these genes. There are two orthologous genes of human $t l r 5$ in zebrafish, $t l r 5 a$ and $t l r 5 b$ and previous studies in our group showed that they are required for activation of host defense genes upon flagellin stimulation. This was shown by simultaneous coknockdown of $t$ tr $5 a$ and $t l r 5 b$ by morpholinos [41]. In this study, $\operatorname{tr} 5 a$ and $t \operatorname{tr} 5 b$ morpholinos were injected separately and, subsequently the morphants were stimulated with flagellin at $27 \mathrm{hpf}$. Embryos treated with standard control morpholino were used as a control [41]. The expression of $i l 1 b$ was measured at 1 hpi by qPCR. Our results revealed that abrogation of both $\operatorname{tr} 5 a$ and $\operatorname{tr} 5 b$ effectively prevented the $i l 1 b$ up-regulation observed in control embryos upon flagellin stimulation (Fig. 2a).

The function of $t l r 2$ in recognition of Pam3CSK4 was tested in the same manner. Our results showed that $t l r 2$ morphants did not exhibit up-regulation of $i l 1 b$ expression upon Pam3CSK4 stimulation, while the control morphants did (Fig. 2b). In contrast, tlr2 morphants stimulated with flagellin, still showed a significant induction of $i l 1 b$ expression (Fig. 2c). 

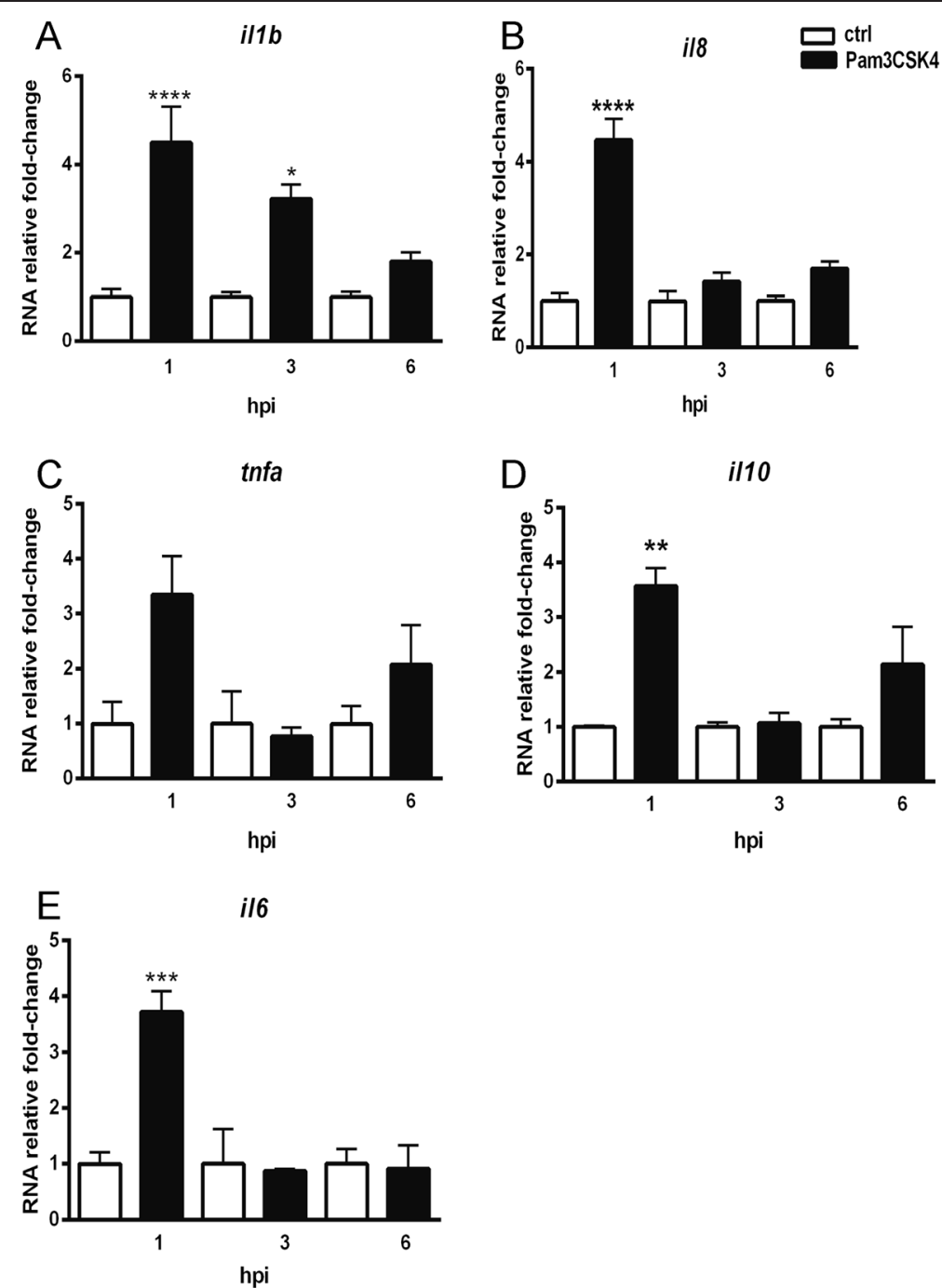

Fig. 1 Immune genes expression at different time points upon Pam3CSK4 stimulation. Embryos were injected at 27 hpf with 1 ng Pam3CSK4 and expression levels of illb (a), il/ (b), tnfa $(\mathbf{c})$, illo (d) and il6 (e) were determined at 1, 3 and 6 hpi by qPCR. Data (mean \pm SEM) are combined from at least three biological replicates ( $n=15$ embryos per group) and expressed relative to their corresponding mock injection (water) control, which is set at 1. Statistical significance of differences between mock and Pam3CSK4 groups was determined by ANOVA analysis and Tukey's multiple comparisons test, ${ }^{*} p<0.05,{ }^{* *} p<0.01,{ }^{* * *} p<0.001$

\section{Identification of a common response gene set for Pam3CSK4 and flagellin stimulation}

Since comparisons of the transcriptome response to PAMPs that activate different TLRs has not been described before in a whole organism we decided to perform RNA deep sequencing (RNAseq) of embryos treated with flagellin and Pam3CSK4 at $1 \mathrm{hpi}$.

Embryos injected with sterile water were used as control and RNA was isolated from a pool of at least fifteen embryos per condition. Triplicates of biological samples were analyzed with Illumina RNAseq and at least 7.2 million mapped reads were obtained for each library (Fig. 3a, Additional file 2: Table S4). Although such reads numbers are insufficient to detect changes in very lowly expressed genes (see Veneman et al, 2014 [49]) approximately 10 million total reads is currently a good cost efficient number that matches the sensitivity of microarrays [50]. The results of the transcriptional responses are summarized in Fig. 3a and Additional file 3: Figure S1. The results show that at any given $p$-value (or false discovery rate-adjusted $p$-value), flagellin leads to a higher number of differentially expressed genes (DEGs) than Pam3CSK4. To further analyze the data, we arbitrarily used a threshold of 2 -fold change and $p$-value $<0.01$. This $p$-value corresponds to FDR adjusted p-values ranging from 0.23 to 0.35 in the different experiments. These selection criteria 

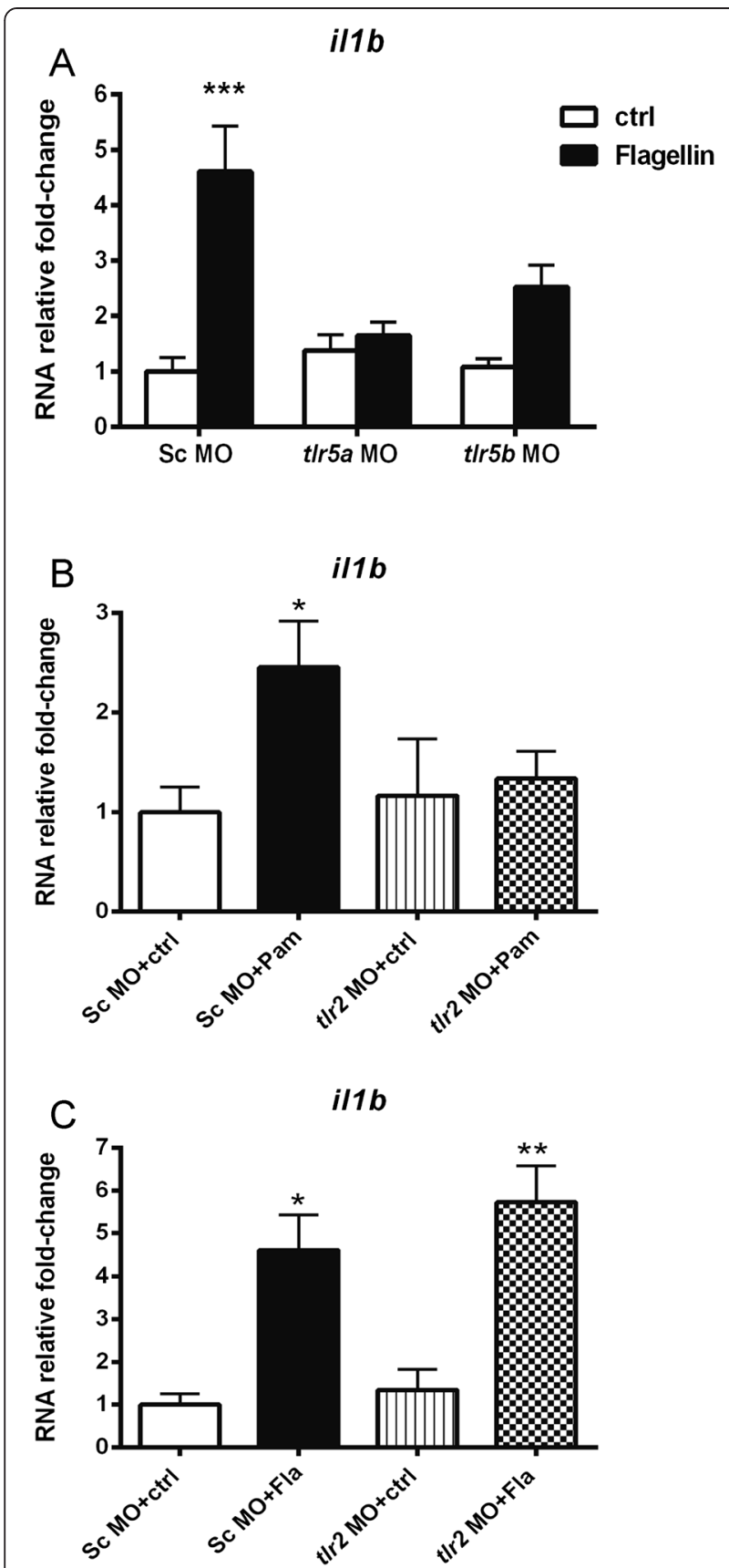

Fig. 2 i/1b expression in t/r2 and t/r5 morphants following PAMPs stimulation. Embryos were injected at the 1-2 cells stage with standard control (Sc), t/r2, t/r5a or t/r5b morpholino (MO) and subsequently injected with Pam3CSK4 at $27 \mathrm{hpf}$, flagellin or water as a mock control. Expression of il1b was determined by qPCR at 1 hpi. a t/r5a and t/r5b knockdown effect on illb RNA expression in response to flagellin. $\mathbf{b} C$ t 1 r2 knockdown effect on il1b RNA expression in response to Pam3CSK4 (b) or flagellin (c). Data (mean \pm SEM) are combined from at least three biological replicates ( $n=10$ embryos per group) and expressed relative to their corresponding water control, which is set at 1. Statistical significance was determined by ANOVA analysis and Tukey's multiple comparisons test, ${ }^{*} p<0.05,{ }^{* *} p<0.01,{ }^{* * *} p<0.001$; Pam, Pam3CSK4 injecton; Fla, flagellin injection are not very stringent so as to prevent loosing genes that are very lowly expressed and therefore with the used sequencing depth will have obtained only low numbers of reads. The entire list of responses without any selection criteria is given in Additional file 4: Table S5 and Additional file 5: Table S6. Applying these settings we obtained 264 DEGs from the Pam3CSK4 stimulated group, composed of 169 up- and 95 down-regulated genes, and 306 DEGs from the flagellin injected group, composed of 180 up- and 126 down-regulated genes (Fig. 3a). Therefore, with both treatments there are more genes upregulated than down-regulated. In the list of top induced and repressed genes there is a lack of any annotation in the data bases (Additional file 4: Table S5 and Additional file 5: Table S6). We compared these two groups of genes and found an overlap set of only 80 genes that include many immune marker genes, such as il1b, tnfb, irak3 and irgll, and transcription factors, like fos, fosl 2 and junba, as shown by the gene ontology terms (GO terms) annotation in Fig. 3b and Additional file 6: Table S3 .

The induction of il1b, tnfa and ils by Pam3CSK4 shown using qPCR (Fig. 1) was confirmed by the RNAseq data (Fig. 3c), but in the case of il6 and il10 there was no induction with flagellin.

\section{Identification of gene sets that are specifically regulated by Pam3CSK4 and flagellin}

This study unexpectedly revealed that there is a relatively large group of genes that are only induced or repressed by either Pam3CSK4 or flagellin (Fig. 3b). To further test whether this specifically induced group is completely unaffected by the other PAMP treatment we used a rigorous filtering approach as shown in Fig. 3d and e. For this approach, the DEGs from the Pam3CSK4 (264 genes) and flagellin (306 genes) stimulation groups were compared with the group of genes that were not affected by flagellin (22611 genes) and Pam3CSK4 (22391 genes), respectively, with a cut-off setting at $<1.4$-fold change and $p>0.05$. By taking the overlap of these sets we thereby exclude genes that were inducible by the other ligand even at very low stringency. This resulted in a set of 48 genes (Fig. 3d) for which the response is specific to Pam3CSK4 and a set of 42 genes (Fig. 3e) for which the response is specific to flagellin. GO analysis indicated that these two groups contain different categories (Fig. 3d, e). Most notably, genes with high fold-change (>2) from the Pam3CSK4 specific group include many transcription factors involved in the TLR signaling pathway, such as junbb, cebpb, fosb, foslla, egr3 and nr4a1. In the flagellin specific group of genes this is not the case and an obvious enriched category could not be identified.

To confirm the result of deep sequencing, qPCR was performed to verify the responses of genes from the Pam3CSK4 specific gene set, namely junbb, cebpb, fosb, 


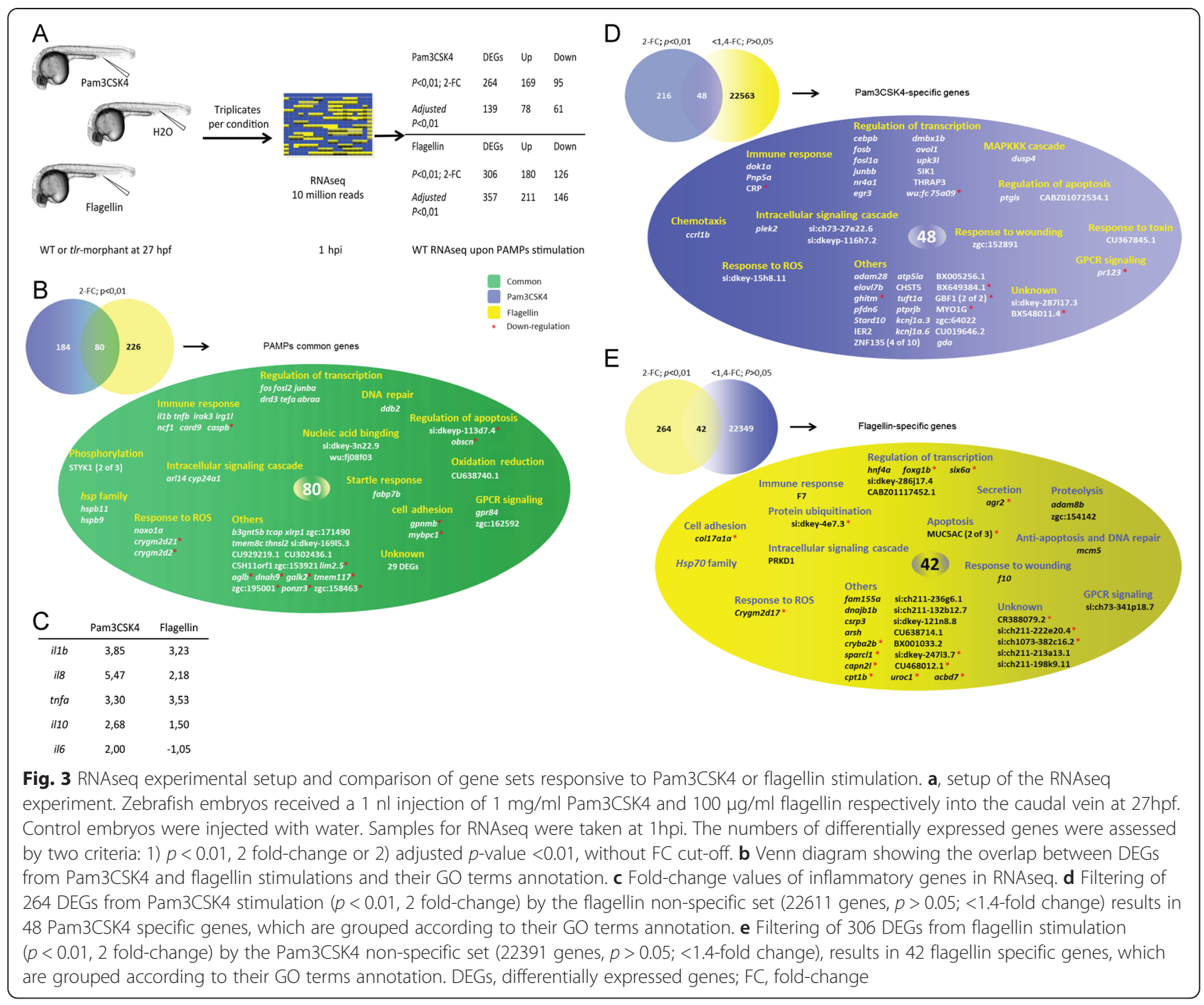

fosl1a, egr3 and $n r 4 a 1$ (Fig. 4). As expected, the expression level of all these transcription factors confirmed the deep sequencing result. Moreover, these genes did not exhibit an apparent differential expression in zebrafish upon flagellin stimulation except fosl1a and junbb (Fig. 4). Even though the expression of these both genes showed significant induction upon flagellin stimulation, the induction level was still far lower than that upon Pam3CSK4 stimulation.

\section{Function of the tIr2 and tlr5a genes in the transcriptome responses to Pam3CSK4 and flagellin}

To confirm that the transcriptome responses upon PAMPs stimulation described above (Figs. 3b and 4) are tlr2 or tlr5 specific, we performed RNAseq analyses of the Pam3CSK4 and flagellin responses under tlr2 and tlr $5 a$ knockdown conditions, again using biological triplicates of each group. Setting a threshold of 2 fold-change and $p<0,01$, we found that the 80 common DEGs responsive to both Pam3CSK 4 and flagellin were reduced by tlr 2 and $t \operatorname{tr} 5 a$ knockdown (Fig. 5c, d and Additional file 6: Table S3). Furthermore, all the 48 genes (40-up and 8-down-regulated) from the Pam3CSK4 specific group showed no longer a differential expression or an anti-correlated expression after tlr2 knockdown and, similarly all the 42 genes (24-up and 18-dow-regulated) from the flagellin specific group showed no longer a differential expression or an anti-correlated expression upon tlr5a abrogation (Fig. 5a, b and Additional file 7: Table S2). Overall, these results confirm the specificity of both morpholinos and show that zebrafish $t l r 2$ and tlr $5 a$ are key mediators of the transcriptomic response triggered by injection of Pam3CSK4 and flagellin respectively.

\section{Discussion and Conclusion}

The signaling pathways underlying recognition of PAMPs have been studied intensively and this has led to a broad 


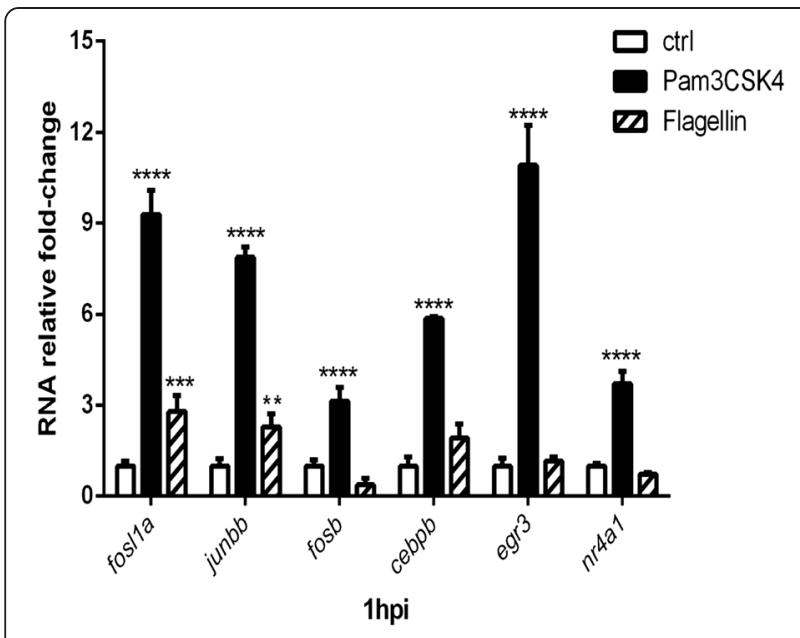

Fig. 4 qPCR analysis of transcription factors genes responsive to PAMPs stimulation. Expression levels of fos $11 a$, fosb, junbb, cebpeb, egr3 and nr4a1 following Pam3CSK4 and flagellin stimulation are determined by qPCR. Data (mean $\pm \mathrm{SD}$ ) are combined from at least tree biological replicates ( $n=15$ embryos per group) and expressed relative to their corresponding water control, which is set at 1. Statistical significance was determined by two-way ANOVA analysis and Tukey's multiple comparisons test, ${ }^{*} p<0.05,{ }^{* *} p<0.01,{ }^{* *} p<0.001,{ }^{* * *} p<0.0001$ understanding of key regulators of innate immunity based on studies of cell cultures and the use of knockout rodent mutants. The new possibilities for analysis of transcriptomes using RNA deep sequencing make it highly attractive to analyze the responses of an entire test animal model at the system biology level. In this manuscript we have chosen the zebrafish embryo model for such an approach and have included functional analysis of Tlr5 and Tlr2 in the response towards two well-known PAMPs, flagellin and Pam3CSK4.

The results show that there is a relatively limited overlap between the transcriptome responses towards flagellin and Pam3CSK4 (Fig. 3b). The overlap includes well known downstream immune mediators that were previously shown to be induced by flagellin [41] such as $i l 1 b$, tnfa, irak3, mmp9, cxcl-clc and ils. In contrast, il6 and il10, that are associated with an anti-inflammatory response, were induced much stronger by Pam3CSK4 than by flagellin. A relatively much larger group of genes showed a differential response to flagellin or Pam3CSK4, including a group of genes of which the transcription is specific for activation by one of the two treatments (Fig. 4).

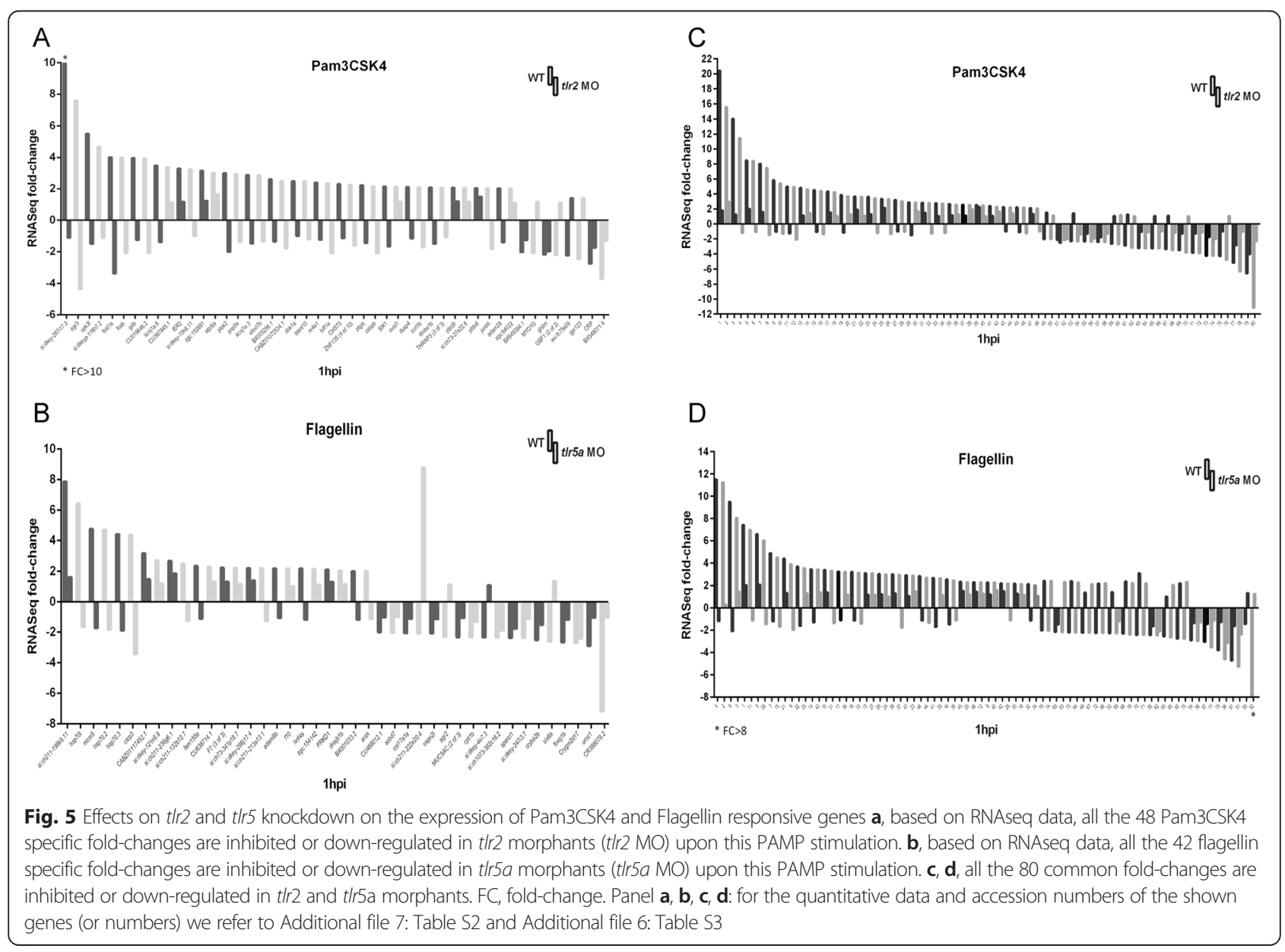


GO terms analysis of the genes specifically regulated by these two PAMPs show that there is an enriched category of transcription factors in the Pam3CSK4-specific group, of which most of genes are up-regulated and only one is down-regulated. Additionally, a less enriched category of immune response genes is found in this group as well, which include a down-regulated CRP (C-reactive protein). For the flagellin-specific group of genes, there are only five genes under the GO-term regulation of transcription of which two are down-regulated. Many of the genes specifically induced by Pam3CSK4 have also been shown to be strongly regulated by infection in the zebrafish embryo system [51] and therefore we would like to further study this group of genes in more detail in future research.

For functional analysis of the transcriptome response towards flagellin and Pam3CSK4 we used morpholinos that were selected on basis of their blocking effect on downstream signaling using qPCR and subsequently confirmed by RNAseq analysis. Surprisingly for $\operatorname{tl} 5$ we found that a morpholino against each of the two copies of this gene, $\operatorname{tr} 5 a$ and $t l r 5 b$ had an effect on downstream signaling, with the tlr5a morpholino giving a complete block of induction of $i l 1 b$ by injection with flagellin and a partial effect of the $t l r 5 b$ morpholino. These data suggests that these two tlr 5 copies function in concert, perhaps by forming heterodimers.

An important question that comes from our work is how to explain the difference in gene sets that are regulated in response to Pam3CSK4 and flagellin? (Fig. 6) Since our detection system seems sufficiently sensitive to detect even minor effects on gene transcription, a limitation in dosing is not a likely explanation for this difference, so instead we think of another two possible alternative explanations. In the first place we could speculate that there are specific downstream signaling partners for Tlr2 and Tlr5. However, such partners have not yet been indicated by previous studies, in contrast, there are evidences that all known direct binding partners are common for both Tlr5 and Tlr2 proteins: including the adaptor proteins Myd88, and Tirap (Mal) that have been implicated in signaling of both proteins [52]. Furthermore, the functions of these genes in the direct recognition of TLR2 and TLR5 ligands have not been tested yet in whole animal models. Mutants for Tirap have not been described yet in zebrafish making the specific function of this gene currently difficult to investigate. Another possible explanation is that the differential response of zebrafish embryos to these two PAMPs is the result of an additive effect of the recognition by different cell types. In this case, the common group of activated downstream genes might be encoded by the response of common immune cells which have a full repertoire of Tlr receptors whereas the specific response might be the result of a distinct transcriptional

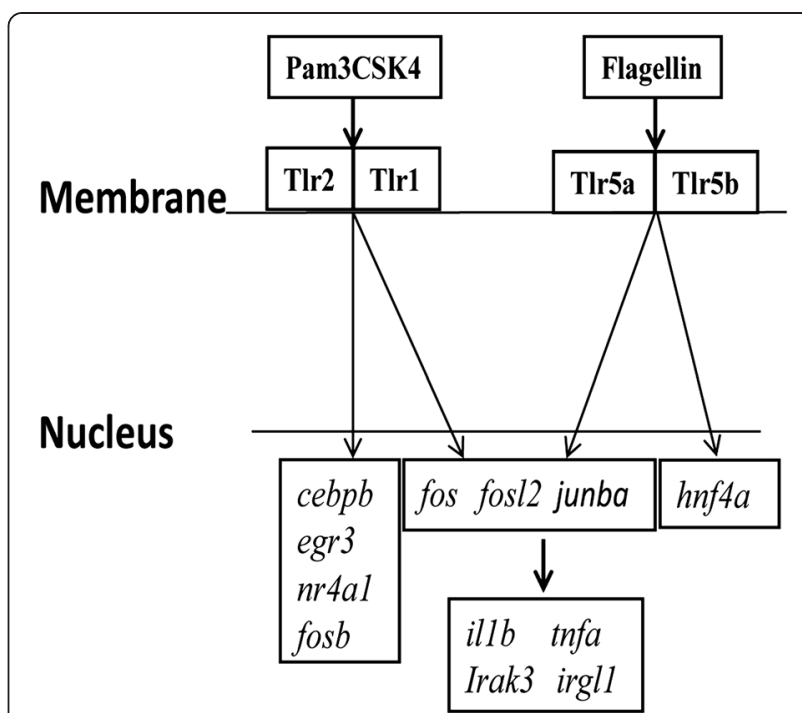

Fig. 6 Specific and common responses to Tlr2 and Tlr5 ligands. The whole organism transcriptome response of zebrafish embryos to treatment with the Tlr2 ligand Pam3CSK4 or the TIr5 ligand flagellin results in the induction of specific and common transcription factor genes as indicated in the figure. The common transcription factor genes (in cooperation with other non-inducible factors, e.g. of the NF-KB family), likely function upstream of the effector genes commonly induced by Pam3CSK4 and flagellin. The transcription factor genes induced by only one of the two ligands are likely to contribute to further specificity in the transcriptional responses of downstream effector genes

response of specialized cells that do not encode all Tlr receptors. The detailed study of these transcription factors will provide valuable information on the specific immune transcriptional signatures elicited by different pathogens. For this purpose the genetic tractability of the zebrafish system will allow the generation of new reporter lines that will contribute to the understanding of how these responses modulate the innate immune system. In addition, such reporter lines will be of general interest since Pam3CSK4 and flagellin signaling pathways are broadly used to study the Tlrs function in inflammatory microbial infection. Furthermore, this signaling pathway is also relevant for studies of atherosclerosis and autoimmune diseases processes $[53,54]$. Therefore the used systemic approach can be highly useful for future studies of a broad spectrum of immune-related diseases.

\section{Additional files}

Additional file 1: Table S1. List of morpholinos and primers.

Additional file 2: Table S4. List of mclusters and mappable reads for Pam3CSK4, flagellin and control RNASeq-library.

Additional file 3: Figure S1. Change trend of the number of DEGs according to different fold-change and $p$-value. 
Additional file 4: Table S5. List of responsive genes to Pam3CSK4 without any selection criteria.

Additional file 5: Table S6. List of responsive genes to flagellin without any selection criteria.

Additional file 6: Table S3. Fold-change and $p$-value of 80 genes responsive to both Pam3CSK4 and flagellin in WT and t/r2- and t/r5a-morphants.

Additional file 7: Table S2. Fold-change and $p$-value of 48 Pam3CSK4- and 42 flagellin-responsive genes in WT and t/r morphants.

\section{Competing interests}

The authors declare that they have no competing interests.

\section{Authors' contributions}

SY carried out all the experimental operations, RNAseq data analysis, and drafted the manuscript. RMJ participated in the design of the study and coordination, helped to draft and revise the final manuscript. AHM helped to draft and revise the final manuscript. HPS conceived the study, participated in its design and coordination, assisted in the data analysis and helped to draft and revise the final manuscript. All authors read and approved the final manuscript.

\section{Acknowledgments}

We thank Ulrike Nehrdich, Davy de Witt and Laura van Hulst for fish caretaking and Hulya Ozupek, Dr. Hans Jansen and Dr. Ron Dirks (ZF-screens B. V.) for assistance with RNAseq analysis. We also want to thank to Dr. Jan de Sonneville and Kees-Jan van der Kolk for making the Genetiles software available.

S. Y. was supported by a grant from the China Scholarship Council (CSC) and R. M. J by the European Marie-Curie Initial Training Network FishForPharma (contract number PITN-GA-2011-289209).

\section{Author details \\ ${ }^{1}$ Institute of Biology, Leiden University, PO Box 95022300 RA Leiden, The Netherlands. ${ }^{2}$ ZF-screens BV, J. H. Oortweg 19, 2333 CH Leiden, The Netherlands. ${ }^{3}$ Present address: Department of Developmental Genetics, Max Planck Institute for Heart and Lung Research, Ludwigstrasse 43, 61231 Bad Nauheim, Germany.}

Received: 11 May 2015 Accepted: 30 June 2015 Published online: 25 July 2015

\section{References}

1. Ulevitch RJ. Molecular mechanisms of innate immunity. Immunol Res. 2000;21(2-3):49-54

2. Masters SL, De Nardo D. Innate immunity. Curr Opin Immunol. 2014;26:v-vi.

3. Wright SD. Toll, a new piece in the puzzle of innate immunity. J Exp Med. 1999;189(4):605-9.

4. Kopp EB, Medzhitov R. The Toll-receptor family and control of innate immunity. Curr Opin Immunol. 1999;11(1):13-8

5. Tan RS, Ho B, Leung BP, Ding JL. TLR cross-talk confers specificity to innate immunity. Int Rev Immunol. 2014;33(6):443-53.

6. Matsumoto M, Funami $K$, Tanabe $M$, Oshiumi $H$, Shingai $M$, Seto $Y$, et al. Subcellular localization of Toll-like receptor 3 in human dendritic cells. J Immunol. 2003;171(6):3154-62.

7. Heil F, Ahmad-Nejad P, Hemmi H, Hochrein H, Ampenberger F, Gellert T, et al. The Toll-like receptor 7 (TLR7)-specific stimulus loxoribine uncovers a strong relationship within the TLR7, 8 and 9 subfamily. Eur J Immunol. 2003;33(11):2987-97.

8. Ahmad-Nejad P, Hacker H, Rutz M, Bauer S, Vabulas RM, Wagner H. Bacterial CpG-DNA and lipopolysaccharides activate Toll-like receptors at distinct cellular compartments. Eur J Immunol. 2002;32(7):1958-68.

9. $\mathrm{Yu} \mathrm{L}$, Wang $\mathrm{L}$, Chen $\mathrm{S}$. Endogenous toll-like receptor ligands and their biological significance. J Cell Mol Med. 2010;14(11):2592-603.

10. Akira S, Takeda K. Toll-like receptor signalling. Nat Rev Immunol. 2004;4(7):499-511.

11. Striz I, Brabcova E, Kolesar L, Sekerkova A. Cytokine networking of innate immunity cells: a potential target of therapy. Clin Sci. 2014;126(9):593-612.
12. Oosting M, Cheng SC, Bolscher JM, Vestering-Stenger R, Plantinga TS, Verschueren IC, et al. Human TLR10 is an anti-inflammatory patternrecognition receptor. Proc Natl Acad Sci U S A. 2014;111(42):E4478-84.

13. Bell JK, Mullen GE, Leifer CA, Mazzoni A, Davies DR, Segal DM. Leucine-rich repeats and pathogen recognition in Toll-like receptors. Trends Immunol. 2003;24(10):528-33.

14. Gong J, Wei T. Structure modeling of Toll-like receptors. Methods Mol Biol. 2014;1169:45-53.

15. Poltorak A, He X, Smirnova I, Liu MY, Van Huffel C, Du X, et al. Defective LPS signaling in $\mathrm{C} 3 \mathrm{H} / \mathrm{HeJ}$ and $\mathrm{C} 57 \mathrm{BL} / 10 \mathrm{ScC}$ mice: mutations in Tlr4 gene. Science. 1998;282(5396):2085-8.

16. Hayashi F, Smith KD, Ozinsky A, Hawn TR, Yi EC, Goodlett DR, et al. The innate immune response to bacterial flagellin is mediated by Toll-like receptor 5. Nature. 2001;410(6832):1099-103.

17. Takeuchi O, Kawai T, Muhlradt PF, Morr M, Radolf JD, Zychlinsky A, et al. Discrimination of bacterial lipoproteins by Toll-like receptor 6 . Int Immunol. 2001;13(7):933-40.

18. Takeuchi O, Sato S, Horiuchi T, Hoshino K, Takeda K, Dong Z, et al. Cutting edge: role of Toll-like receptor 1 in mediating immune response to microbial lipoproteins. J Immunol. 2002;169(1):10-4.

19. Bafica A, Scanga CA, Feng CG, Leifer C, Cheever A, Sher A. TLR9 regulates Th1 responses and cooperates with TLR2 in mediating optimal resistance to Mycobacterium tuberculosis. J Exp Med. 2005;202(12):1715-24.

20. Ding A, Yu H, Yang J, Shi S, Ehrt S. Induction of macrophage-derived SLPI by Mycobacterium tuberculosis depends on TLR2 but not MyD88. Immunology. 2005;116(3):381-9.

21. McBride A, Konowich J, Salgame P. Host defense and recruitment of Foxp3(+) T regulatory cells to the lungs in chronic Mycobacterium tuberculosis infection requires toll-like receptor 2. Plos Pathog. 2013;9(6):e1003397. doi:10.1371/journal.ppat.1003397.

22. Drage MG, Tsai HC, Pecora ND, Cheng TY, Arida AR, Shukla S, et al. Mycobacterium tuberculosis lipoprotein LprG (Rv1411c) binds triacylated glycolipid agonists of Toll-like receptor 2. Nat Struct Mol Biol. 2010;17(9):1088-95. doi:10.1038/nsmb.1869.

23. Drage MG, Pecora ND, Hise AG, Febbraio M, Silverstein RL, Golenbock DT, et al. TLR2 and its co-receptors determine responses of macrophages and dendritic cells to lipoproteins of Mycobacterium tuberculosis. Cell Immunol. 2009;258(1):29-37. doi:10.1016/j.cellimm.2009.1003.1008.

24. Means TK, Wang S, Lien E, Yoshimura A, Golenbock DT, Fenton MJ. Human toll-like receptors mediate cellular activation by Mycobacterium tuberculosis. J Immunol. 1999;163(7):3920-7.

25. Kanczkowski W, Morawietz H, Ziegler CG, Funk RH, Schmitz G, Zacharowski K, et al. Pam3CSK4 and LTA-TLRs ligands associated with microdomains induce IL8 production in human adrenocortical cancer cells. Horm Metab Res. 2007;39(6):457-60.

26. Lombardi V, Van Overtvelt L, Horiot S, Moussu H, Chabre H, Louise A, et al. Toll-like receptor 2 agonist Pam3CSK4 enhances the induction of antigen-specific tolerance via the sublingual route. Clin Exp Allergy. 2008;38(11):1819-29.

27. Mintz M, Mintz D, Ilia-Ezra R, Shpigel NY. Pam3CSK4/TLR2 signaling elicits neutrophil recruitment and restricts invasion of Escherichia coli P4 into mammary gland epithelial cells in a murine mastitis model. Vet Immunol Immunopathol. 2013;152(1-2):168-75.

28. Agrawal S, Gollapudi S, Gupta S, Agrawal A. Dendritic cells from the elderly display an intrinsic defect in the production of $\mathrm{LL}-10$ in response to lithium chloride. Exp Gerontol. 2013;48(11):1285-92.

29. Cabanski M, Wilhelm J, Zaslona Z, Steinmuller M, Fink L, Seeger W, et al. Genome-wide transcriptional profiling of mononuclear phagocytes recruited to mouse lungs in response to alveolar challenge with the TLR2 agonist Pam3CSK4. Am J Physiol Lung Cell Mol Physiol. 2009;297(4):L608-18.

30. Stridh L, Ek CJ, Wang X, Nilsson H, Mallard C. Regulation of Toll-like receptors in the choroid plexus in the immature brain after systemic inflammatory stimuli. Translational Stroke Res. 2013;4(2):220-7.

31. Willett CE, Cortes A, Zuasti A, Zapata AG. Early hematopoiesis and developing lymphoid organs in the zebrafish. Dev Dyn. 1999;214(4):323-36.

32. Davidson AJ, Zon LI. The 'definitive' (and 'primitive') guide to zebrafish hematopoiesis. Oncogene. 2004;23(43):7233-46.

33. Lam SH, Chua HL, Gong Z, Lam TJ, Sin YM. Development and maturation of the immune system in zebrafish, Danio rerio: a gene expression profiling, in situ hybridization and immunological study. Dev Comp Immunol. 2004;28(1):9-28 
34. van der Vaart M, van Soest JJ, Spaink HP, Meijer AH. Functional analysis of a zebrafish myd88 mutant identifies key transcriptional components of the innate immune system. Dis Model Mech. 2013;6(3):841-54.

35. Liu Y, Li M, Fan S, Lin Y, Lin B, Luo F, et al. A unique feature of Toll/IL-1 receptor domain-containing adaptor protein is partially responsible for lipopolysaccharide insensitivity in zebrafish with a highly conserved function of MyD88. J Immunol. 2010;185(6):3391-400.

36. Meijer AH, Gabby Krens SF, Medina Rodriguez IA, He S, Bitter W, Ewa Snaar-Jagalska B, et al. Expression analysis of the Toll-like receptor and TIR domain adaptor families of zebrafish. Mol Immunol. 2004;40(11):773-83.

37. Fan S, Chen S, Liu Y, Lin Y, Liu H, Guo L, et al. Zebrafish TRIF, a Golgilocalized protein, participates in IFN induction and NF-kappaB activation. J Immunol. 2008;180(8):5373-83.

38. Jault C, Pichon L, Chluba J. Toll-like receptor gene family and TIR-domain adapters in Danio rerio. Mol Immunol. 2004;40(11):759-71.

39. Stockhammer OW, Rauwerda H, Wittink FR, Breit TM, Meijer AH, Spaink HP. Transcriptome analysis of Traf6 function in the innate immune response of zebrafish embryos. Mol Immunol. 2010;48(1-3):179-90.

40. Phelan PE, Mellon MT, Kim CH. Functional characterization of full-length TLR3, IRAK-4, and TRAF6 in zebrafish (Danio rerio). Mol Immunol. 2005;42(9):1057-71.

41. Stockhammer OW, Zakrzewska A, Hegedus Z, Spaink HP, Meijer AH. Transcriptome profiling and functional analyses of the zebrafish embryonic innate immune response to Salmonella infection. J Immunol. 2009;182(9):5641-53.

42. van Soest JJ, Stockhammer OW, Ordas A, Bloemberg GV, Spaink HP, Meijer $\mathrm{AH}$. Comparison of static immersion and intravenous injection systems for exposure of zebrafish embryos to the natural pathogen Edwardsiella tarda. BMC Immunol. 2011;12:58.

43. Marin-Juez R, Jong-Raadsen S, Yang S, Spaink HP. Hyperinsulinemia induces insulin resistance and immune suppression via Ptpn6/Shp1 in zebrafish. J Endocrinol. 2014;222(2):229-41.

44. Langmead B, Salzberg SL. Fast gapped-read alignment with Bowtie 2. Nat Methods. 2012;9(4):357-9.

45. Li H, Handsaker B, Wysoker A, Fennell T, Ruan J, Homer N, et al. The sequence alignment/Map format and SAMtools. Bioinformatics. 2009;25(16):2078-9.

46. Anders $\mathrm{S}$, Reyes A, Huber W. Detecting differential usage of exons from RNA-seq data. Genet Res. 2012;22(10):2008-17.

47. Anders $\mathrm{S}, \mathrm{Huber}$ W. Differential expression analysis for sequence count data. Genome Biol. 2010;11(10):R106.

48. Huang DW, Sherman BT, Lempicki RA. Systematic and integrative analysis of large gene lists using DAVID bioinformatics resources. Nat Protocols. 2008;4(1):44-57.

49. Veneman WJ, de Sonneville J, van der Kolk KJ, Ordas A, Al-Ars Z, Meijer AH, et al. Analysis of RNAseq datasets from a comparative infectious disease zebrafish model using GeneTiles bioinformatics. Immunogenetics. 2014;67(3):135-47.

50. Ballouz S, Verleyen W, Gillis J. Guidance for RNA-seq co-expression network construction and analysis: safety in numbers. Bioinformatics. 2015;31(13):2123-30

51. Veneman WJ, Stockhammer OW, de Boer L, Zaat SA, Meijer AH, Spaink HP. A zebrafish high throughput screening system used for Staphylococcus epidermidis infection marker discovery. BMC Genomics. 2013;14:255.

52. Choi YJ, Jung J, Chung HK, Im E, Rhee SH. PTEN regulates TLR5-induced intestinal inflammation by controlling Mal/TIRAP recruitment. Faseb J. 2013;27(1):243-54.

53. Bielinski SJ, Hall JL, Pankow JS, Boerwinkle E, Matijevic-Aleksic N, He M, et al. Genetic variants in TLR2 and TLR4 are associated with markers of monocyte activation: the atherosclerosis risk in communities MRI study. Hum Genet. 2011;129(6):655-62.

54. Fang J, Fang D, Silver PB, Wen F, Li B, Ren X, et al. The role of TLR2, TRL3, TRL4, and TRL9 signaling in the pathogenesis of autoimmune disease in a retinal autoimmunity model. Invest Ophthalmol Vis Sci. 2010;51(6):3092-9.

\section{Submit your next manuscript to BioMed Central and take full advantage of:}

- Convenient online submission

- Thorough peer review

- No space constraints or color figure charges

- Immediate publication on acceptance

- Inclusion in PubMed, CAS, Scopus and Google Scholar

- Research which is freely available for redistribution

Submit your manuscript at www.biomedcentral.com/submit 